\title{
Constructing Rule-based Solvers for Intentionally-defined Constraints
}

\author{
Ingi Sobhi ${ }^{1}$, Slim Abdennadher ${ }^{1}$, and Hariolf Betz ${ }^{2}$ \\ 1 Faculty of Media Engineering and Technology, German University in Cairo, Egypt \\ [Slim.Abdennadher, Ingi.Sobhi] @guc .edu.eg \\ 2 Faculty of Engineering and Computer Science, University of Ulm, Germany \\ Hariolf.Betz@uni-ulm.de
}

\begin{abstract}
Developing constraint solvers which are key requisites of constraint programming languages is time consuming and difficult. In this paper, we propose a generic algorithm that symbolically constructs rulebased solvers from the intensional definition of the constraint. Unlike the well-established "generate and test" approach, our symbolic construction approach is capable of generating recursive rules from a recursive constraint definition. Combining the two approaches gives better filtering capabilities than either of the approaches acting alone.
\end{abstract}

\section{Introduction}

"Constraint Programming represents one of the closest approaches computer science has yet made to the Holy Grail of programming: the user states the problem, the computer solves it." [E. Freuder]

The validity of this statement for a Constraint Logic Programming (CLP) language is contingent on the existence of constraint solvers. These associate constraints with filtering algorithms that remove variable values which cannot belong to any solution of the problem.

Constraint Handling Rules (CHR) is a multi-headed guarded and concurrent constraint logic programming language. To incorporate constraint solvers in CHR, a scheme was proposed in [1] to automatically derive the solvers given the intentional definition of the constraints. The scheme is based on a generate and test approach where rule candidates are enumerated and tested for validity against the constraint definition. Although the approach performs an extensive search for valid rules, given a recursive constraint definition it is unable to generate recursive rules.

To overcome this, we propose a scheme where valid rules are symbolically constructed from the clauses of a CLP program defining the constraint. The idea behind the construction stems from the observation that if in a non-overlapping CLP program the execution of a clause leads to a solution, the execution of all other clauses will not. Thus our constructed CHR rules simplify the constraint to the body of a clause only if all other clauses do not hold. Moreover, we combine the two schemes to achieve better filtering. 
Example 1 (Motivation). Consider the lexicographic order constraint [2-4]. Given two sequences $L_{1}$ and $L_{2}$ of variables of the same length, then lex holds if $L_{1}$ is lexicographically smaller than or equal to $L_{2}$. The following CLP program defines the lex $\left(L_{1}, L_{2}\right)$ constraint:

$$
\begin{aligned}
& \operatorname{lex}([],[]) \\
& \operatorname{lex}\left(\left[X_{1} \mid T_{1}\right],\left[X_{2} \mid T_{2}\right]\right) \leftarrow X_{1}<X_{2} \\
& \operatorname{lex}\left(\left[X_{3} \mid T_{3}\right],\left[X_{4} \mid T_{4}\right]\right) \leftarrow X_{3}=X_{4} \wedge \operatorname{lex}\left(T_{3}, T_{4}\right)
\end{aligned}
$$

The generate and test approach [1] generates rules that reason about the first elements of the two lists such as:

$$
\operatorname{lex}\left(L_{1}, L_{2}\right) \Rightarrow L_{1}=\left[X_{1} \mid T_{1}\right] \wedge L_{2}=\left[X_{2} \mid T_{2}\right] \mid X_{1} \leq X_{2}
$$

The symbolic construction approach proposed in this paper generates the following solver:

$$
\begin{aligned}
& \operatorname{lex}\left(L_{1}, L_{2}\right) \Leftrightarrow L_{1}=[] \vee L_{2}=[] \mid L_{1}=[] \wedge L_{2}=[] \\
& \operatorname{lex}\left(L_{1}, L_{2}\right) \Leftrightarrow L_{1}=\left[X_{1} \mid T_{1}\right] \wedge L_{2}=\left[X_{2} \mid T_{2}\right] \wedge X_{1} \neq X_{2} \mid X_{1}<X_{2} \\
& \operatorname{lex}\left(L_{1}, L_{2}\right) \Leftrightarrow L_{1}=\left[X_{1} \mid T_{1}\right] \wedge L_{2}=\left[X_{2} \mid T_{2}\right] \wedge X_{1} \geq X_{2} \mid X_{1}=X_{2} \wedge \operatorname{lex}\left(T_{1}, T_{2}\right)
\end{aligned}
$$

Given the query $\left\langle\operatorname{lex}\left(\left[A_{1}, A_{2}, A_{3}\right],\left[B_{1}, B_{2}, B_{3}\right]\right)\right\rangle$ where the domains of the variables are defined as follows:

$$
\begin{aligned}
A_{1}=\{1,3,4\}, A_{2}=\{2,3,4\}, A_{3} & =\{1,2\} \\
B_{1}=\{1\}, \quad B_{2}=\{2\}, \quad B_{3} & =\{0,1,2\}
\end{aligned}
$$

The generate and test approach enforces the constraint $A_{1} \leq B_{1}$, which removes the values $\{3,4\}$ from the domain of $A_{1}$. The solution becomes:

$$
\begin{aligned}
& A_{1}=\{1\}, A_{2}=\{2,3,4\}, A_{3}=\{1,2\}, \\
& B_{1}=\{1\}, \quad B_{2}=\{2\}, \quad B_{3}=\{0,1,2\}, \\
& \quad \operatorname{lex}\left(\left[A_{1}, A_{2}, A_{3}\right],\left[B_{1}, B_{2}, B_{3}\right]\right)
\end{aligned}
$$

For the symbolic construction approach since $A_{1} \geq B_{1}$, rule (4) is executed enforcing equality on the values of $A_{1}$ and $B_{1}$ before calling lex recursively on the remaining list elements. Since $A_{2} \geq B_{2}$ rule (4) is applied again, whereas for $A_{3}$ and $B_{3}$ no rule is applicable. The solution becomes:

$$
\begin{gathered}
A_{1}=\{1\}, A_{2}=\{2\}, A_{3}=\{1,2\}, \\
B_{1}=\{1\}, B_{2}=\{2\}, B_{3}=\{0,1,2\}, \\
\operatorname{lex}\left(\left[A_{3}\right],\left[B_{3}\right]\right)
\end{gathered}
$$

Combining both approaches prunes the domains of the variables further since rule (1) is applicable to $\operatorname{lex}\left(\left[A_{3}\right],\left[B_{3}\right]\right)$ and filters the domain of $B_{3}$. The combined solution becomes:

$$
\begin{gathered}
A_{1}=\{1\}, A_{2}=\{2\}, A_{3}=\{1,2\}, \\
B_{1}=\{1\}, B_{2}=\{2\}, B_{3}=\{1,2\}, \\
A_{3} \leq B_{3}, \operatorname{lex}\left(\left[A_{3}\right],\left[B_{3}\right]\right)
\end{gathered}
$$

We will proceed with the lex constraint in all examples of this paper. 
The paper is a revised and extended version of [5] and is organized as follows. In section 3, we present the symbolic construction approach and prove soundness and termination of the constructed solvers. In section 4, we apply post-processing methods to improve the run-time complexity of the solvers. Finally, section 5 combines the symbolic construction approach with the "generate and test" approach to achieve better filtering.

\section{Preliminaries}

\subsection{Intentional Definition}

Let $p$ be a constraint. A CLP program $P$ defines $p$ if $p$ occurs with the same arity in the head of all the clauses and all true instances of $p$ are accounted for (closed world assumption). The program $P$ is of the usual form:

$$
p\left(\bar{t}_{1}\right) \leftarrow C_{1}, p\left(\bar{t}_{2}\right) \leftarrow C_{2}, \ldots, p\left(\bar{t}_{n}\right) \leftarrow C_{n}
$$

where $\bar{t}_{i}$ stands for a sequence of terms and $C_{i}$ is a conjunction of built-in and user-defined constraints. Built-in constraints are those defined by a constraint theory and for which solvers are available. These solvers are assumed to be well-behaved (terminating and confluent), closed under negation, and achieve arc-consistency. User-defined constraints are those for which solvers are needed. The symbolic construction approach requires that there are no variables in $C_{i}$ that are not in $\bar{t}_{i}$ and that all clauses are non-overlapping (i.e. in a computation at most one clause can lead to a solution).

Definition 1. The logical reading of $P$ denoted by $P^{*}$ is given by its Clark completion [6]:

$$
\forall \bar{x}\left(p(\bar{x}) \leftrightarrow \bigvee_{i=1}^{n} \exists \bar{y}_{i}\left(\bar{x}=\bar{t}_{i} \wedge C_{i}\right)\right)
$$

where $\bar{x}$ is a sequence of distinct fresh variables and $\bar{y}_{i}$ is the sequence of variables in $\bar{t}_{i}$. The expression $\bar{x}=\bar{t}_{i}$ stands for the conjunction of equations between respective elements of the sequences $\bar{x}$ and $\bar{t}_{i}$.

Example 2 (Clark Completion). The Clark completion of the CLP program defining lex is:

$$
\begin{aligned}
\forall L_{1}, L_{2} \operatorname{lex} & \left(L_{1}, L_{2}\right) \leftrightarrow \\
& \left(L_{1}=[] \wedge L_{2}=[]\right) \vee \\
& \exists X_{1}, X_{2}, T_{1}, T_{2}\left(L_{1}=\left[X_{1} \mid T_{1}\right] \wedge L_{2}=\left[X_{2} \mid T_{2}\right] \wedge X_{1}<X_{2}\right) \vee \\
& \exists X_{3}, X_{4}, T_{3}, T_{4}\left(L_{1}=\left[X_{3} \mid T_{3}\right] \wedge L_{2}=\left[X_{4} \mid T_{4}\right] \wedge X_{3}=X_{4} \wedge \operatorname{lex}\left(T_{3}, T_{4}\right)\right)
\end{aligned}
$$




\subsection{Constraint Solver}

CHR [7] specifies how new constraints interact with the constraint store and is thus especially suited for writing constraint solvers. It has two main rule types:

$$
\begin{array}{ll}
\text { Simplification Rule: } & H \Leftrightarrow G \mid B \\
\text { Propagation Rule: } & H \Rightarrow G \mid B
\end{array}
$$

where the head $H$ are user-defined constraints, the guard $G$ are built-in constraints and the body constraints $B$ are both.

Definition 2. The logical meaning of a simplification rule is a logical equivalence provided the guard holds:

$$
\forall \bar{x} \forall \bar{y}(G \rightarrow(H \leftrightarrow \exists \bar{z} B))
$$

where $\bar{x}$ is the set of variables occurring in $H$, the variables $\bar{y}$ are the set occurring in $G$ but not in $H$ and $\bar{z}$ are the variables occurring in $B$ only. Similarly, the logical meaning of a propagation rule is an implication provided the guard holds.

Prompted with a query, applicable rules are executed until a fixpoint is reached where no more rules can be applied or a contradiction occurs. A rule is applicable provided that constraints from the query match the head and imply the guard. Execution of a simplification rule rewrites constraints that match the head by the body while execution of a propagation rule adds the body constraints to the constraint store.

\subsection{Generate and Test Approach}

In this section, we summarize the generate and test approach presented in [1]. Given a CLP program defining the constraint as well as the syntactic form of the candidate rules defined by the following sets:

- Baselhs contains constraints that must appear in the head of all rules,

- Candlhs contains constraints to be used in conjunction with Base lhs $_{\text {s }}$ to form the head, and

- Cand $_{r h s}$ contains constraints that may appear in the body.

The generate and test approach generates valid rules as follows. Candidate propagation rules of the form $H \Rightarrow B$ are enumerated, and subjected to a validity test based on the observation that a rule is valid if the execution of the goal $H \wedge \neg(B)$ finitely fails with respect to the CLP program.

Example 3 (Generate and Test Approach). Given the syntactic form of candidate rules for lex as:

$$
\begin{aligned}
& \text { Base }_{\text {lhs }}=\left\{\operatorname{lex}\left(L_{1}, L_{2}\right)\right\} \\
& \text { Cand }_{l h s}=\left\{L_{1}=[], L_{2}=[], L_{1}=\left[X_{1} \mid T_{1}\right], L_{2}=\left[X_{2} \mid T_{2}\right], X_{1} \leq X_{2}, X_{1}>X_{2}, X_{1}<X_{2}, \ldots\right\} \\
& \text { Cand }_{r h s}=\text { Cand }_{l h s}
\end{aligned}
$$


The generate and test approach generates (among others) the following rule for lex:

$$
\operatorname{lex}\left(\left[X_{1} \mid T_{1}\right],\left[X_{2} \mid T_{2}\right]\right) \Rightarrow X_{1} \leq X_{2}
$$

The rule is generated since executing the goal $\left\langle\operatorname{lex}\left(\left[X_{1} \mid T_{1}\right],\left[X_{2} \mid T_{2}\right]\right), X_{1}>X_{2}\right\rangle$ fails as demonstrated by the following derivation tree:

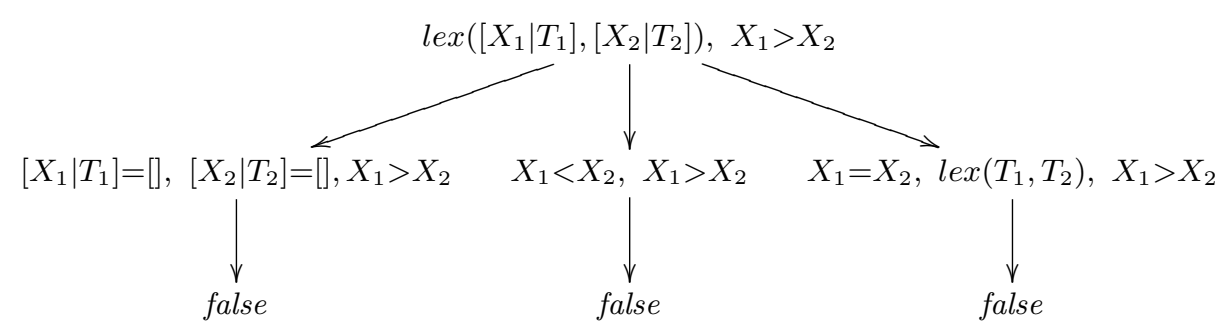

\section{Symbolic Construction Approach}

The symbolic construction approach (Fig. 1) constructs a solver for a constraint by symbolically transforming the Clark completion of the CLP program defining the constraint to semantically valid rules. The idea of the transformation stems from the observation that in a non-overlapping CLP program if the execution of one clause leads to a solution then the execution of all other clauses will not. Thus to construct a rule that simplifies the constraint to the body of one clause, the negation of the bodies of all other clauses is added to the guard. This ensures that the rule is applicable only when all other clauses are not valid and hence maintains consistency with the constraint definition.

begin

$p$ : left hand side of the Clark completion

Disjuncts: set of disjuncts of right hand side of the Clark completion

Rules $=\{\}$ : resultant rule set

for each $D$ in Disjuncts do

Other $=$ Disjuncts $\backslash\{D\}$

Rules $=$ Rules $\cup\{p \Leftrightarrow \neg$ Other $\mid D\}$

end for

end

Fig. 1. Symbolic Construction Algorithm 


\subsection{Guard Determination}

More formally, given the definition of a constraint $p(\bar{x})$ :

$$
\forall \bar{x}\left(p(\bar{x}) \leftrightarrow \bigvee_{i=1}^{n} \exists \bar{y}_{i}\left(\bar{x}=\bar{t}_{i} \wedge C_{i}\right)\right)
$$

The symbolic construction algorithm constructs rules of the form:

$$
p(\bar{x}) \Leftrightarrow \neg \bigvee_{j=1, j \neq i}^{n} \exists \bar{y}_{j}\left(\bar{x}=\bar{t}_{j} \wedge C_{j}\right) \mid \bar{x}=\bar{t}_{i} \wedge C_{i} \quad \text { for each } i \in\{1, \ldots, n\}
$$

where $\bar{x}=\bar{t}_{j}$ stands for the conjunction of equations between respective elements of the sequences $\bar{x}$ and $\bar{t}_{j}$. According to the soundness proof (given in the next section), this is equivalent to:

$$
p(\bar{x}) \Leftrightarrow \bigwedge_{j=1, j \neq i}^{n} \forall \bar{y}_{j}\left(\bar{x} \neq \bar{t}_{j}\right) \vee \exists \bar{y}_{j}\left(\bar{x}=\bar{t}_{j} \wedge \neg C_{j}\right) \mid \bar{x}=\bar{t}_{i} \wedge C_{i}
$$

where $\bar{x} \neq \bar{t}_{j}$ stands for the disjunction of negated equations between respective elements of the sequences $\bar{x}$ and $\bar{t}_{j}$ and $\neg C_{j}$ is a disjunction of negated constraints. The symbolic construction approach distinguishes between the two cases for negated constraints: Negated built-ins are replaced by the corresponding positive constraints since built-ins are closed under negation. Negated userdefined constraints are discarded and constructing an entailment checker that determines if a user-defined constraint does not hold is left for future work. Thus, the general form of the rules is:

$$
p(\bar{x}) \Leftrightarrow \bigwedge_{j=1, j \neq i}^{n} \bigvee_{k=1}^{|x|+m_{j}} E_{j}^{k} \mid \bar{x}=\bar{t}_{i} \wedge C_{i}
$$

where

$$
\begin{array}{cc}
E_{j}^{k}=\forall \bar{y}_{j}^{k}\left(x^{k} \neq t_{j}^{k}\right) & \text { for } k \in\{1, \ldots,|x|\}, \\
E_{j}^{|x|+k}=\exists \bar{y}_{j}\left(\bar{x}=\bar{t}_{j} \wedge \neg c_{j}^{k}\right) & \text { for } k \in\left\{1, \ldots, m_{j}\right\},
\end{array}
$$

the $\bar{y}_{j}^{k}$ is the sequence of variables in the term $t_{j}^{k}$ and $m_{j}$ is the number of built-in constraints in $C_{j}$.

Example 4 (Symbolic Construction Approach). The lex constraint has three disjuncts namely:

$$
\begin{array}{ll}
D_{1}: & L_{1}=[] \wedge L_{2}=[] \\
D_{2}: & \exists X_{1}, X_{2}, T_{1}, T_{2}\left(L_{1}=\left[X_{1} \mid T_{1}\right] \wedge L_{2}=\left[X_{2} \mid T_{2}\right] \wedge X_{1}<X_{2}\right) \\
D_{3}: & \exists X_{3}, X_{4}, T_{3}, T_{4}\left(L_{1}=\left[X_{3} \mid T_{3}\right] \wedge L_{2}=\left[X_{4} \mid T_{4}\right] \wedge X_{3}=X_{4} \wedge \operatorname{lex}\left(T_{3}, T_{4}\right)\right)
\end{array}
$$


To construct a rule that simplifies lex to the first disjunct, the negation of the other two disjuncts is added to the guard:

$$
\begin{aligned}
& \neg\left(\exists X_{1}, X_{2}, T_{1}, T_{2}\left(L_{1}=\left[X_{1} \mid T_{1}\right] \wedge L_{2}=\left[X_{2} \mid T_{2}\right] \wedge X_{1}<X_{2}\right) \vee\right. \\
& \left.\quad \exists X_{3}, X_{4}, T_{3}, T_{4}\left(L_{1}=\left[X_{3} \mid T_{3}\right] \wedge L_{2}=\left[X_{4} \mid T_{4}\right] \wedge X_{3}=X_{4} \wedge \operatorname{lex}\left(T_{3}, T_{4}\right)\right)\right)
\end{aligned}
$$

This is equivalent to:

$$
\begin{aligned}
&\left(\forall X_{1}, T_{1}\left(L_{1} \neq\left[X_{1} \mid T_{1}\right]\right)\right. \vee \forall X_{2}, T_{2}\left(L_{2} \neq\left[X_{2} \mid T_{2}\right]\right) \vee \\
&\left.\exists X_{1}, X_{2}, T_{1}, T_{2}\left(L_{1}=\left[X_{1} \mid T_{1}\right] \wedge L_{2}=\left[X_{2} \mid T_{2}\right] \wedge X_{1} \geq X_{2}\right)\right) \wedge \\
&\left(\forall X_{3}, T_{3}\left(L_{1} \neq\left[X_{3} \mid T_{3}\right]\right) \vee \forall X_{4}, T_{4}\left(L_{2} \neq\left[X_{4} \mid T_{4}\right]\right) \vee\right. \\
&\left.\exists X_{3}, X_{4}, T_{3}, T_{4}\left(L_{1}=\left[X_{3} \mid T_{3}\right] \wedge L_{2}=\left[X_{4} \mid T_{4}\right] \wedge X_{3} \neq X_{4}\right)\right)
\end{aligned}
$$

where negated built-ins are replaced by the corresponding positive constraints and negated user-defined constraints discarded.

Since the arguments of lex are (ordered) lists, the constraint $\forall X, T L \neq[X \mid T]$ can be simplified to $L=[$. The expression becomes:

$$
\begin{aligned}
& \left(L_{1}=[] \vee L_{2}=[] \vee\left(L_{1}=\left[X_{1} \mid T_{1}\right] \wedge L_{2}=\left[X_{2} \mid T_{2}\right] \wedge X_{1} \geq X_{2}\right)\right) \wedge \\
& \left(L_{1}=[] \vee L_{2}=[] \vee\left(L_{1}=\left[X_{3} \mid T_{3}\right] \wedge L_{2}=\left[X_{4} \mid T_{4}\right] \wedge X_{3} \neq X_{4}\right)\right)
\end{aligned}
$$

Thus the constructed rule is:

$$
\begin{aligned}
\operatorname{lex}\left(L_{1}, L_{2}\right) \Leftrightarrow & \\
& \left(L_{1}=[] \vee L_{2}=[] \vee\left(L_{1}=\left[X_{1} \mid T_{1}\right] \wedge L_{2}=\left[X_{2} \mid T_{2}\right] \wedge X_{1} \geq X_{2}\right)\right) \wedge \\
& \left(L_{1}=[] \vee L_{2}=[] \vee\left(L_{1}=\left[X_{3} \mid T_{3}\right] \wedge L_{2}=\left[X_{4} \mid T_{4}\right] \wedge X_{3} \neq X_{4}\right)\right) \\
& \mid L_{1}=[] \wedge L_{2}=[]
\end{aligned}
$$

\subsection{The Solver Properties}

In this section we prove soundness and termination as well as discuss completeness of the constructed solvers.

Soundness. A simplification rule $H \Leftrightarrow G \mid B$ is valid w.r.t. a CLP program $P$ and the constraint theory $C T$ iff $P^{*} \cup C T \models \forall \bar{x}(\exists \bar{y} G \rightarrow(H \leftrightarrow \exists \bar{z} B))$.

Theorem 1 (Soundness). The symbolic construction algorithm constructs valid simplification rules w.r.t. the CLP program and the constraint theory.

Proof (Soundness). Consider Clark's completion of the CLP program defining a constraint $p$ :

$$
\forall \bar{x}\left(p(\bar{x}) \leftrightarrow\left(\bigvee_{i=1}^{n} \exists \bar{y}_{i}\left(\bar{x}=\bar{t}_{i} \wedge C_{i}\right)\right)\right)
$$


For every $i \in\{1, \ldots, n\}$, we therefore have:

$$
\forall \bar{x}\left(\left(\neg \bigvee_{j=1, j \neq i}^{n} \exists \bar{y}_{j}\left(\bar{x}=\bar{t}_{j} \wedge C_{j}\right)\right) \rightarrow\left(p(\bar{x}) \leftrightarrow \exists \bar{y}_{i}\left(\bar{x}=\bar{t}_{i} \wedge C_{i}\right)\right)\right)
$$

and consequently:

$$
\forall \bar{x}\left(\left(\bigwedge_{j=1, j \neq i}^{n} \neg \exists \bar{y}_{j}\left(\bar{x}=\bar{t}_{j} \wedge C_{j}\right)\right) \rightarrow\left(p(\bar{x}) \leftrightarrow \exists \bar{y}_{i}\left(\bar{x}=\bar{t}_{i} \wedge C_{i}\right)\right)\right)
$$

which is the logical reading of a CHR rule:

$$
p(\bar{x}) \Leftrightarrow G \mid \bar{x}=\bar{t}_{i} \wedge C_{i}
$$

where $G$ is equivalent to:

$$
\bigwedge_{j=1, j \neq i}^{n} \neg \exists \bar{y}_{j}\left(\bar{x}=\bar{t}_{j} \wedge C_{j}\right)
$$

Recall that $\bar{y}_{j}$ denotes the variables in $\bar{t}_{j}$ and that $\bar{y}_{j}$ is disjoint from $\bar{x}$. Therefore, for every $j \in\{1, \ldots, n\}$ and every valuation of $\bar{x}$ such that $\bar{x}=\bar{t}_{j}$ is satisfiable, there exists a sequence of terms $\bar{u}_{j}$ such that:

$$
\left(\bar{x}=\bar{t}_{j}\right) \Leftrightarrow\left(\bar{y}_{j}=\bar{u}_{j}\right)
$$

This observation guarantees the existence of a function $u_{j}$ for each $j \in$ $\{1, \ldots, n\}$ that maps from sequences of terms to sequences of terms such that:

$$
\left(\exists \bar{y}_{j} \bar{x}=\bar{t}_{j}\right) \Rightarrow\left(\left(\bar{x}=\bar{t}_{j}\right) \Leftrightarrow\left(\bar{y}_{j}=u_{j}(\bar{x})\right)\right)
$$

and consequently:

$$
\left(\bar{x}=\bar{t}_{j}\right) \Rightarrow\left(\bar{y}_{j}=u_{j}(\bar{x})\right)
$$

Using function $u_{j}$, we have that:

$$
\neg \exists \bar{y}_{j} \quad\left(\bar{x}=\bar{t}_{j} \wedge C_{j}\right)
$$

is equivalent to:

$$
\neg \exists \bar{y}_{j} \quad\left(\bar{x}=\bar{t}_{j} \wedge \bar{y}_{j}=u_{j}(\bar{x}) \wedge C_{j}\right)
$$

From there, the substitution property of equality gives us:

$$
\neg \exists \bar{y}_{j} \quad\left(\bar{x}=\bar{t}_{j} \wedge C_{j}\left[\bar{y}_{j} / u_{j}(\bar{x})\right]\right)
$$

We move the negation to the inside of the formula and get:

$$
\forall \bar{y}_{j} \quad\left(\bar{x} \neq \bar{t}_{j} \vee \neg C_{j}\left[\bar{y}_{j} / u_{j}(\bar{x})\right]\right)
$$


As the variables $\bar{y}_{j}$ do not appear in the formula $\neg C_{j}\left[\bar{y}_{j} / u_{j}(\bar{x})\right]$, we can move it outside of the universal quantification:

$$
\forall \bar{y}_{j}\left(\bar{x} \neq \bar{t}_{j}\right) \vee \neg C_{j}\left[\bar{y}_{j} / u_{j}(\bar{x})\right]
$$

Applying $(A \vee B) \Leftrightarrow(A \vee(\neg A \wedge B))$ gives us:

$$
\forall \bar{y}_{j}\left(\bar{x} \neq \bar{t}_{j}\right) \vee\left(\exists \bar{y}_{j}\left(\bar{x}=\bar{t}_{j}\right) \wedge \neg C_{j}\left[\bar{y}_{j} / u_{j}(\bar{x})\right]\right)
$$

As the variables $\bar{y}_{j}$ do not appear in the formula $\neg C_{j}\left[\bar{y}_{j} / u_{j}(\bar{x})\right]$, we can move it into the scope of their existential quantification:

$$
\forall \bar{y}_{j}\left(\bar{x} \neq \bar{t}_{j}\right) \vee \exists y_{j}\left(\bar{x}=\bar{t}_{j} \wedge \neg C_{j}\left[\bar{y}_{j} / u_{j}(\bar{x})\right]\right)
$$

According to the definition of the function $u_{j}, \bar{x}=\bar{t}_{j}$ implies $\bar{y}_{j}=u_{j}(\bar{x})$ :

$$
\forall \bar{y}_{j}\left(\bar{x} \neq \bar{t}_{j}\right) \vee \exists y_{j}\left(\bar{x}=\bar{t}_{j} \wedge \bar{y}_{j}=u_{j}(\bar{x}) \wedge \neg C_{j}\left[\bar{y}_{j} / u_{j}(\bar{x})\right]\right)
$$

We apply the substitution property of equality again to get:

$$
\forall \bar{y}_{j}\left(\bar{x} \neq \bar{t}_{j}\right) \vee \exists y_{j}\left(\bar{x}=\bar{t}_{j} \wedge \bar{y}_{j}=u_{j}(\bar{x}) \wedge \neg C_{j}\right)
$$

As $\left(\bar{x}=\bar{t}_{j}\right) \Rightarrow\left(\bar{y}_{j}=u_{j}(\bar{x})\right)$, this is equivalent to:

$$
\forall \bar{y}_{j} \quad\left(\bar{x} \neq \bar{t}_{j}\right) \vee \exists y_{j}\left(\bar{x}=\bar{t}_{j} \wedge \neg C_{j}\right)
$$

Therefore, the guard $G$ of the generated CHR rule is equivalent to:

$$
\left(\bigwedge_{j=1, j \neq i}^{n}\left(\forall \bar{y}_{j}\left(\bar{x} \neq \bar{t}_{j}\right) \vee \exists y_{j}\left(\bar{x}=\bar{t}_{j} \wedge \neg C_{j}\right)\right)\right)
$$

Termination. In [8] proving the termination of CHR solvers is based on polynomial interpretations where the rank of a term or an atom is defined by a linear positive combination of the rankings of its arguments. The basic idea is to prove that the rank of the head of a rule is strictly larger than that of its body. Moreover, built-in solvers are assumed to be well-behaved (terminating and confluent) and thus the rank of built-in constraints is defined as 0 .

Theorem 2 (Termination). For constraints defined by a CLP program where the rank of the head of every clause is strictly larger than that of its body, the symbolic construction approach constructs terminating solvers.

Proof (Termination). If for each clause of the CLP program, the rank of the head of the clause is strictly larger than that of its body, then the constructed solver terminates. The head and body of a constructed rule are the same as the clause and only built-in constraints which are defined as 0 are added to the guard. Thus the head of a constructed rule is strictly larger than that of its body and the solver terminates. 
Completeness. The constructed solvers can not guarantee propagation completeness for non-trivial constraints since negated user-defined constraints are ignored.

\section{Solver Optimization}

To improve the runtime efficiency of the solvers and readability of the rules, redundant guard entailment checks are removed. This is achieved by expanding the guard expressions to disjunctive normal form and splitting each disjunct into a new rule. Then, we apply the redundant rules removal algorithm of [9] on the complete rule set. After redundant rules are removed, guards originating from the same rule are recombined to avoid loss of completeness.

\subsection{Guard Splitting}

The symbolic construction approach constructs rules of the form:

$$
p(\bar{x}) \Leftrightarrow \bigwedge_{j=1, j \neq i}^{n} \bigvee_{k=1}^{|x|+m_{j}} E_{j}^{k} \mid \bar{x}=\bar{t}_{i} \wedge C_{i}
$$

where

$$
\begin{array}{ll}
E_{j}^{k}=\forall \bar{y}_{j}^{k}\left(x^{k} \neq t_{j}^{k}\right) & \text { for } k \in\{1, \ldots,|x|\}, \\
E_{j}^{|x|+k}=\exists \bar{y}_{j}\left(\bar{x}^{\mid x} \bar{t}_{j} \wedge \neg c_{j}^{k}\right) & \text { for } k \in\left\{1, \ldots, m_{j}\right\},
\end{array}
$$

the $\bar{y}_{j}^{k}$ is the sequence of variables in the term $t_{j}^{k}$ and $m_{j}$ is the number of built-in constraints in $C_{j}$.

To split the guard into rules (Fig. 2), we distribute the conjunction over the disjunction and get a formula in disjunctive normal form where the number of disjuncts is $\Pi_{j=1, j \neq i}^{n}|x|+m_{j}$. Then each disjunct is simplified to an equivalent conjunction of constraints by the available built-in solver and superfluous disjuncts removed. These include multiple occurrences of a disjunct (irrespective of the order of constraints within the disjunct) and false. Each simplified disjunct is split into a new rule.

Example 5 (Guard Splitting). Consider the previously constructed rule of lex:

$$
\begin{aligned}
\operatorname{lex}\left(L_{1}, L_{2}\right) \Leftrightarrow & \\
& \left(L_{1}=[] \vee L_{2}=[] \vee\left(L_{1}=\left[X_{1} \mid T_{1}\right] \wedge L_{2}=\left[X_{2} \mid T_{2}\right] \wedge X_{1} \geq X_{2}\right)\right) \wedge \\
& \left(L_{1}=[] \vee L_{2}=[] \vee\left(L_{1}=\left[X_{3} \mid T_{3}\right] \wedge L_{2}=\left[X_{4} \mid T_{4}\right] \wedge X_{3} \neq X_{4}\right)\right) \\
& \mid L_{1}=[] \wedge L_{2}=[]
\end{aligned}
$$




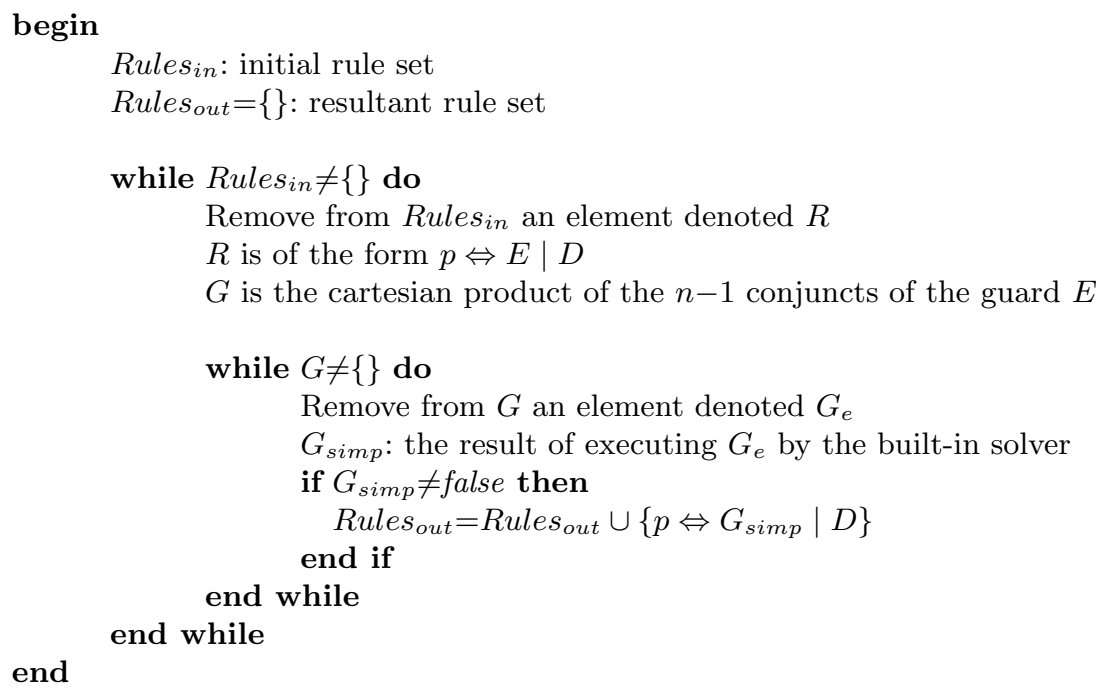

Fig. 2. Guard Splitting

Transforming the guard expression to disjunctive normal form, we get:

$$
\begin{aligned}
& \left(L_{1}=[] \wedge L_{1}=[]\right) \vee \\
& \left(L_{1}=[] \wedge L_{2}=[]\right) \vee \\
& \left(L_{1}=[] \wedge L_{1}=\left[X_{3} \mid T_{3}\right] \wedge L_{2}=\left[X_{4} \mid T_{4}\right] \wedge X_{3} \neq X_{4}\right) \vee \\
& \left(L_{2}=[] \wedge L_{1}=[]\right) \vee \\
& \left(L_{2}=[] \wedge L_{2}=[]\right) \vee \\
& \left(L_{2}=[] \wedge L_{1}=\left[X_{3} \mid T_{3}\right] \wedge L_{2}=\left[X_{4} \mid T_{4}\right] \wedge X_{3} \neq X_{4}\right) \vee \\
& \left(L_{1}=\left[X_{1} \mid T_{1}\right] \wedge L_{2}=\left[X_{2} \mid T_{2}\right] \wedge X_{1} \geq X_{2} \wedge L_{1}=[]\right) \vee \\
& \left(L_{1}=\left[X_{1} \mid T_{1}\right] \wedge L_{2}=\left[X_{2} \mid T_{2}\right] \wedge X_{1} \geq X_{2} \wedge L_{2}=[]\right) \vee \\
& \left(L_{1}=\left[X_{1} \mid T_{1}\right] \wedge L_{2}=\left[X_{2} \mid T_{2}\right] \wedge X_{1} \geq X_{2} \wedge L_{1}=\left[X_{3} \mid T_{3}\right] \wedge L_{2}=\left[X_{4} \mid T_{4}\right] \wedge X_{3} \neq X_{4}\right)
\end{aligned}
$$

To simplify the resultant expression, each disjunct is executed by the built-in constraints solver and superfluous disjuncts removed. We assume that for the conjunction of constraints, the built-in solver:

- Removes identical occurrences of constraints

- Simplifies constraints (e.g. $L=[] \wedge L=[X \mid T] \Leftrightarrow$ false and $X \geq Y \wedge X \neq Y \Leftrightarrow X>Y$ )

- Propagates new constraints (e.g. $L_{1}=\left[X_{1} \mid T_{1}\right] \wedge L_{2}=\left[X_{2} \mid T_{2}\right] \wedge L_{1}=\left[X_{3} \mid T_{3}\right] \wedge$ $\left.L_{2}=\left[X_{4} \mid T_{4}\right] \Rightarrow X_{1}=X_{3} \wedge T_{1}=T_{3} \wedge X_{2}=X_{4} \wedge T_{2}=T_{4}\right)$

The expression simplifies to:

$$
\left(L_{1}=[]\right) \vee\left(L_{1}=[] \wedge L_{2}=[]\right) \vee\left(L_{2}=[]\right) \vee\left(L_{1}=\left[X_{1} \mid T_{1}\right] \wedge L_{2}=\left[X_{2} \mid T_{2}\right] \wedge X_{1}>X_{2}\right)
$$


which splits into the following rules:

$$
\begin{aligned}
& \operatorname{lex}\left(L_{1}, L_{2}\right) \Leftrightarrow L_{1}=[] \mid L_{1}=[] \wedge L_{2}=[] \\
& \operatorname{lex}\left(L_{1}, L_{2}\right) \Leftrightarrow L_{1}=[] \wedge L_{2}=[] \mid L_{1}=[] \wedge L_{2}=[] \\
& \operatorname{lex}\left(L_{1}, L_{2}\right) \Leftrightarrow L_{2}=[] \mid L_{1}=[] \wedge L_{2}=[] \\
& \operatorname{lex}\left(L_{1}, L_{2}\right) \Leftrightarrow L_{1}=\left[X_{1} \mid T_{1}\right] \wedge L_{2}=\left[X_{2} \mid T_{2}\right] \wedge X_{1}>X_{2} \mid L_{1}=[] \wedge L_{2}=[]
\end{aligned}
$$

\subsection{Redundant Rules Removal}

To remove redundant rules, the algorithm of [9] is used. The idea of the algorithm is based on operational equivalence of programs. The algorithm (Fig. 3) basically checks if the computation step due to a rule can be performed by the remainder of the program. It determines this by executing the head and guard in both the program and the program without the rule in it. If the results are identical upto renaming of variables and logical equivalence of built-in constraints), then the rule is obviously redundant and can be removed.

\section{begin}

Rules $_{\text {in }}$ : the initial rule set

Rules $_{\text {out }}$ : the resultant rule set without redundancy

Rules $_{\text {out }}=$ Rules $_{\text {in }}$

while Rules $_{\text {in }} \neq\{\}$ do

Remove from Rules $_{i n}$ an element denoted $R$

lhs: the head and guard of the rule $R$

$S_{1}$ : the result of executing lhs in Rules out

Rules $_{\text {remaining }}=$ Rules $_{\text {out }} \backslash\{R\}$

$S_{2}$ : the result of executing lhs in Rules remaining $_{1}$

if $S_{1}$ is identical to $S_{2}$ then

$$
\text { Rules }_{\text {out }}=\text { Rules }_{\text {remaining }}
$$

end while

$$
\text { end if }
$$

end

Fig. 3. Redundancy Removal Algorithm

Example 6 (Redundant Rules Removal). Consider the following two rules of lex:

$$
\begin{aligned}
& \operatorname{lex}\left(L_{1}, L_{2}\right) \Leftrightarrow L_{1}=[] \mid L_{1}=[] \wedge L_{2}=[] \\
& \operatorname{lex}\left(L_{1}, L_{2}\right) \Leftrightarrow L_{1}=[] \wedge L_{2}=[] \mid L_{1}=[] \wedge L_{2}=[]
\end{aligned}
$$

The second rule is redundant since its operation is covered by the first rule. Removing the second rule from the rule set and querying the remaining set with 
its head and guard $\langle A=[] \wedge B=[] \wedge \operatorname{lex}(A, B)\rangle$, the first rule is applied and gives the same result $\langle A=[] \wedge B=[]\rangle$ as the second rule.

After redundant rules are removed, guards originating from the same rule are recombined to avoid loss of completeness. Further guard optimization techniques have been addressed in [10].

\section{Combined Approach}

To improve the filtering capabilities of our constructed solvers, we propose extending our solvers with rules generated by the orthogonal approach "generate and test" of [1]. To reduce the search space of the generate and test method, the symbolic construction algorithm is run first and the constructed rules eliminated from the enumeration tree of the generate and test. Moreover, the algorithm of [9] is used to remove the redundant rules of the combined solver. In general, the combined solvers are more expressive than the solvers of either approaches.

Example 7 (Combined Approach). The combined solver for lex is given below. The first three rules represent the solver obtained from the symbolic construction approach and the last rule is added by the generate and test.

$$
\begin{aligned}
\operatorname{lex}\left(L_{1}, L_{2}\right) \Leftrightarrow & L_{1}=[] \vee L_{2}=[] \mid L_{1}=[] \wedge L_{2}=[] \\
\operatorname{lex}\left(L_{1}, L_{2}\right) \Leftrightarrow & L_{1}=\left[X_{3} \mid T_{3}\right] \wedge L_{2}=\left[X_{4} \mid T_{4}\right] \wedge X_{3} \neq X_{4} \mid \\
& L_{1}=\left[X_{1} \mid T_{1}\right] \wedge L_{2}=\left[X_{2} \mid T_{2}\right] \wedge X_{1}<X_{2} \\
\operatorname{lex}\left(L_{1}, L_{2}\right) \Leftrightarrow & L_{1}=\left[X_{1} \mid T_{1}\right] \wedge L_{2}=\left[X_{2} \mid T_{2}\right] \wedge X_{1} \geq X_{2} \mid \\
& L_{1}=\left[X_{3} \mid T_{3}\right] \wedge L_{2}=\left[X_{4} \mid T_{4}\right] \wedge X_{3}=X_{4} \wedge \operatorname{lex}\left(T_{3}, T_{4}\right) \\
\operatorname{lex}\left(\left[X_{1} \mid T_{1}\right],\left[X_{2} \mid T_{2}\right]\right) \Rightarrow & X_{1} \leq X_{2}
\end{aligned}
$$

The solver is sound. All rules are logical consequences of the constraint definition.

The solver terminates. The interesting case for termination is the recursive rule (3). The ranking function for $\operatorname{lex}\left(L_{1}, L_{2}\right)$ is defined as the positive combination of the rank of its arguments:

$$
\operatorname{rank}\left(\operatorname{lex}\left(L_{1}, L_{2}\right)\right)=\operatorname{length}\left(L_{1}\right)+\operatorname{length}\left(L_{2}\right)
$$

The length of a list is expressed in the ranking function scheme as:

$$
\begin{aligned}
\text { length }([]) & =0 \\
\text { length }([H \mid T]) & =1+\text { length }(T)
\end{aligned}
$$

All other constraints in the rule are built-ins and are ranked as 0 . The rule terminates since the rank of the head and guard is greater than that of its body:

$$
\operatorname{rank}\left(\operatorname{lex}\left(\left[X_{1} \mid T_{1}\right],\left[X_{2} \mid T_{2}\right]\right)\right)>\operatorname{rank}\left(\operatorname{lex}\left(T_{1}, T_{2}\right)\right)
$$


The solver is not propagation complete. In [4] the below complete lex solver was presented:

$$
\begin{aligned}
\operatorname{lex}([],[]) & \Leftrightarrow \operatorname{true} \\
\operatorname{lex}\left(\left[X_{1} \mid T_{1}\right],\left[X_{2} \mid T_{2}\right]\right) \Leftrightarrow & X_{1}<X_{2} \mid \operatorname{true} \\
\operatorname{lex}\left(\left[X_{1} \mid T_{1}\right],\left[X_{2} \mid T_{2}\right]\right) \Leftrightarrow & X_{1}=X_{2} \mid \operatorname{lex}\left(T_{1}, T_{2}\right) \\
\operatorname{lex}\left(\left[X_{1} \mid T_{1}\right],\left[X_{2} \mid T_{2}\right]\right) \Rightarrow & X_{1} \leq X_{2} \\
\operatorname{lex}\left(\left[X_{1}, U \mid T_{1}\right],\left[X_{2}, V \mid T_{2}\right]\right) \Leftrightarrow & U>V \mid X_{1}<X_{2} \\
\operatorname{lex}\left(\left[X_{1}, U \mid T_{1}\right],\left[X_{2}, V \mid T_{2}\right]\right) \Leftrightarrow & U \geq V \wedge T_{1}=[-\mid] \mid \\
& \operatorname{lex}\left(\left[X_{1}, U\right],\left[X_{2}, V\right]\right) \wedge \operatorname{lex}\left(\left[X_{1} \mid T_{1}\right],\left[X_{2} \mid T_{2}\right]\right)
\end{aligned}
$$

The solver consists of three pairs of rules: the first two correspond to base cases of the recursion, the middle two perform forward reasoning, and the last two perform backward reasoning. By comparison we find that the backward reasoning rules are not subsumed by our combined lex solver rendering the solver incomplete.

Consider the query $\left\langle\operatorname{lex}\left(\left[A_{1}, A_{2}, A_{3}, A_{4}\right],\left[B_{1}, B_{2}, B_{3}, B_{4}\right]\right)\right\rangle$ where the domains of the variables are defined as follows:

$$
\begin{array}{r}
A_{1}=\{1,3,4\}, A_{2}=\{1,2,3,4,5\}, A_{3}=\{1,2\}, A_{4}=\{3,4,5\} \\
B_{1}=\{1\}, B_{2}=\{0,1,2,3,4\}, B_{3}=\{0,1\}, B_{4}=\{0,1,2\}
\end{array}
$$

In the case of the combined solver for lex, rule (3) is fired since $A_{1} \geq B_{1}$ enforcing equality on the values of $A_{1}$ and $B_{1}$ before calling lex recursively on the remaining list elements. The relation between $A_{2}$ and $B_{2}$ satisfies none of the guards, thus rule (4) is fired which enforces $A_{2} \leq B_{2}$. The solution becomes:

$$
\begin{array}{r}
A_{1}=\{1\}, A_{2}=\{1,2,3,4\}, A_{3}=\{1,2\}, A_{4}=\{3,4,5\}, \\
B_{1}=\{1\}, B_{2}=\{1,2,3,4\}, B_{3}=\{0,1\}, B_{4}=\{0,1,2\}, \\
A_{2} \leq B_{2}, \operatorname{lex}\left(\left[A_{2}, A_{3}, A_{4}\right],\left[B_{2}, B_{3}, B_{4}\right]\right)
\end{array}
$$

In the case of the lex solver of [4], rules (8), (7), (10), and (9) are applied in that order and further constrain the domains of the variables to:

$$
\begin{array}{r}
A_{1}=\{1\}, A_{2}=\{1,2,3\}, A_{3}=\{1,2\}, A_{4}=\{3,4,5\}, \\
B_{1}=\{1\}, B_{2}=\{2,3,4\}, B_{3}=\{0,1\}, B_{4}=\{0,1,2\}, \\
A_{2}<B_{2}, \operatorname{lex}\left(\left[A_{2}, A_{3}\right],\left[B_{2}, B_{3}\right]\right)
\end{array}
$$

\section{Conclusion}

In this paper we have presented an algorithm that automatically constructs rule-based solvers from the constraint definition. The algorithm is an orthogonal approach to the general direction of the work done in the field as it is based on symbolic construction rather than a generate and test method. Contrary to 
other approaches, given a recursive constraint definition the algorithm is able to generate recursive rules which allow reasoning over arguments of arbitrary length.

The constructed solvers are a good basis for constraint reasoning and can be extended manually or with rules generated using other approaches. We have proposed extending our rules with those generated by the algorithm in [1]. In general, the solvers generated using the combined approach are more expressive than those generated by either of the two approaches acting alone.

An interesting direction for future work to improve the expressiveness of the generated solvers is to incorporate negated user-defined constraints in the symbolic construction approach.

Acknowledgments. We would like to thank Thom Frühwirth, Frank Raiser, Jon Sneyers and the anonymous reviewers for valuable comments on a preliminary version of this paper.

\section{References}

1. Abdennadher, S., Rigotti, C.: Automatic Generation of CHR Constraint Solvers. Journal of Theory and Practice of Logic Programming (TPLP) 5(4-5) (2005) 403418

2. Carlsson, M., Beldiceanu, N.: Revisiting the Lexicographic Ordering Constraint. In: Technical Report T2002-17. Swedish Institute of Computer Science (2002)

3. Frisch, A., Hnich, B., Kzltan, Z., Miguel, I., Walsh, T.: Global Constraints for Lexicographic Orderings. In: International Conference on Principles and Practice of Constraint Programming, CP2002. Volume 2470 of Lecture Notes in Computer Science., Springer (2002) 93-108

4. Frühwirth, T.: Complete Propagation Rules for Lexicograhic Order Constraints over Arbitary Domains. In: Recent Advances in CHR. Volume 3978 of Lecture Notes in Computer Science., Springer (2006) 14-28

5. Abdennadher, S., Sobhi, I.: Generation of Rule-based Constraint Solvers: Combined Approach. In: International Symposium in Logic-Based Program Synthesis and Transformation, LOPSTR07, Revised Selected Papers. Volume 4915 of Lecture Notes in Computer Science., Springer (2008) 106-120

6. Clark, K.: Negation as Failure. In: Logic and Databases. Plenum Press, New York (1978) 293-322

7. Frühwirth, T.: Theory and Practice of Constraint Handling Rules, Special Issue on Constraint Logic Programming. Journal of Logic Programming 37(1-3) (1998) 95-138

8. Frühwirth, T.: Proving Termination of Constraint Solver Programs. In: New Trends in Constraints. Volume 1865 of Lecture Notes in Computer Science., Springer (2000) $298-317$

9. Abdennadher, S., Frühwirth, T.: Integration and Optimization of Rule-based Constraint Solvers. In: International Symposium on Logic-based Program Synthesis and Transformation, LOPSTR03. Volume 3018 of Lecture Notes in Computer Science., Springer (2004) 198-213

10. Sneyers, J., Schrijvers, T., Demoen, B.: Guard and Continuation Optimization for Occurrence Representations of CHR. In: International Conference on Logic Programming, ICLP05. Volume 3668 of Lecture Notes in Computer Science., Springer (2005) 83-97 